DOI: $10.2478 /$ jec-2019-0001

\title{
ENHANCING THE VALUE OF LIFESTYLE BUNDLES
}

\author{
Michael Levens ${ }^{1}$ \\ ${ }^{1}$ Walsh College, Troy, MI, USA, mlevens@walshcollege.edu
}

\begin{abstract}
Research purpose. The study of marketing a lifestyle offering involves allowing brands from different categories to collaborate in giving, essentially, a new offering as a bundle to consumers. This is particularly important for brands operating in segments that have a significant loyalty or in segments that have become or are becoming commodities. A lifestyle offering can span categories and address latent consumer attitudes such as aspiration and convenience. Lifestyle bundles were introduced as a "way of life that individuals express by choosing to allocate their time and personal resources to a single package of two or more goods or services that they believe represent their current or desired way of life" (Levens, 2010). The initial research on lifestyle bundles was contextualized through a bundled multi-category luxury offering, targeting affluent consumers, which was built on the concepts of luxury marketing, bundling and the needs, attitudes and behaviours of affluent consumers. The bundle items include a home, a vehicle and a vacation club. This paper revisits the original work on this topic and explores ways to enhance interest in the underlying bundle proposition.
\end{abstract}

Design / Methodology / Approach. The methodology to explore enhancements to the lifestyle bundles involved re-analyzing the data cube used to develop the inaugural lifestyle bundle research (Levens, 2010). The data cube was mined to identify individual preferences to increase consumer interest in the original concept. The underlying argument is that adjusting the lifestyle bundle items may increase consumer interest in the overall concept. The analysis presented in this paper was completed through statistical description using SPSS software.

Findings. The initial concept evaluation identified a non-trivial level of interest in the lifestyle bundle consisting of a home, a vehicle and a vacation club. This paper identifies modifications to the bundles that would increase consumer interest among rejecters and supporters of the lifestyle bundle concept. While novelty was acknowledged as a reason for consumer interest, it was clear that utility derived from convenience and lifestyle expression was the basis for many consumers supporting the concept. Concept rejecters also noted these attributes as influencing their opinions. Elimination of the vacation club offer from the lifestyle bundle would have the greatest impact on increasing rejection. Adding home and vehicle insurance to the lifestyle bundle would have the greatest impact on enhancing the value proposition for concept supporters.

Originality / Value / Practical implications. Lifestyle bundles are a novel approach for marketers to differentiate their products and create new opportunities among consumers who might have not previously considered their products or services. Enhancing the lifestyle bundle offer can only increase those opportunities.

Keywords: Conjoint research; Lifestyle bundles.

JEL code: M31.

\section{Introduction}

The study of marketing a lifestyle offering involves allowing brands from different categories to collaborate to give, essentially, a new offering as a bundle to consumers. This is particularly important for brands operating in segments with significant loyalty or in segments that have become or are becoming commodities. A lifestyle offering can span categories and address latent consumer attitudes such as aspiration and convenience. Lifestyle bundles were introduced as a "way of life that individuals express by choosing to allocate their time and personal resources to a single package of two or more goods or services that they believe represent their current or desired way of life" (Levens, 2010). The initial research on lifestyle bundles was contextualized through a bundled multi-category luxury offering, targeting affluent consumers, which was built on the concepts of luxury marketing, bundling and the needs, attitudes and behaviours of affluent consumers. The bundle items include a home, a 
vehicle and a vacation club. This paper revisits the original work on this topic and explores ways to enhance consumer interest in the underlying bundle proposition.

\section{Literature Review}

The increasing competition between brands and commoditization of many market categories provided the impetus for original work on lifestyle bundles (Levens, 2010). The concept of bundling and its influence on consumer behavior provided the framework for constructing and testing the original bundles. That relationship also provides context when considering enhancements to lifestyle bundles.

Differentiation remains a strategic approach when attempting to increase perceived value for a particular target. Primary strategies to achieve differentiation include innovation, enhancing customer relationships and bundling (Freeman et al., 2004). Bundling represents the idea that a combined offering of products is perceived to offer greater value to consumers than when the individual components of the bundle are sold independently (Levens, 2016). When that combined offering spans different market categories, purchase decisions can be linked, which would influence consumer behavior (Shocker et al., 2004).

Utility theory, the idea that consumers will consider all available information in a comprehensive manner when making purchase decisions, is considered the foundation for many econometric models and is quite commonly mentioned in the bundling literature (Warsh, 1989). Gaeth et al. (1990) argue that consumers evaluate bundles by averaging individual utilities of each item within the bundle. However, bundling research introduces other consumer behavior factors that must be considered. Oppenwal \& Holyoake (2004) note that specific bundles can reduce search and transaction costs and that consumers often want that a pre-developed bundle should "fit" together. Gwin and Gwin (2003) contribute the idea that products should be considered a collection of attributes and that consumers select products and services based on those attributes.

Research into lifestyle bundles was designed to explore the interaction between bundling and consumer behavior. Lifestyle marketing, previously referenced in the literature either as a marketing communication theme or marketing segmentation variable, was selected as the platform to build lifestyle bundles (Levens, 2010). The question posed was “can the lifestyle itself be the product?" Levens (2010) found that there was non-trivial interest in the lifestyle bundle and that convenience was a significant factor in generating utility. Now that the question has been answered affirmatively that lifestyle itself can be the product, the next question is "can the lifestyle product be enhanced?"

\section{Methodology}

The methodology to explore enhancements to the lifestyle bundles involved re-analyzing the data cube used to develop the inaugural lifestyle bundle research (Levens, 2010). The data cube was mined to identify individual preferences to increase consumer interest in the original concept. The underlying argument is that adjusting (removing, adding or replacing) lifestyle bundle items may increase overall interest in the concept.

The concept evaluation statement was as follows:

A new concept in lifestyle marketing: A single luxury offer purchased at one time consisting of a home, a vehicle, and a vacation club designed specifically to fit your needs, wants and desires. Whether you prefer to lease or buy, there are numerous financing options that span timeframes between 3 and 30 years. The process would begin with an in-person consultation with a trained lifestyle consultant that would involve listening to your interests and desires in addition to administering a set of specific questions to help identify the best lifestyle fit. A personalized profile would be developed by the lifestyle consultant and prepared for your consideration.

\section{Design}

The original data set was created through an online survey research including screener questions and a confidentiality agreement for the concept test. The main question architecture covered ownership profile, purchase horizon, attitudinal questions, concept exposure through a choice scenario and concept 
purchase interest factors and non-interest factors as well as classification questions. The total number of exposure questions, depending on skip patterns, typically exceeded 60. Lifestyle bundle utility was generated using choice-based conjoint analysis (Raghavarao et al., 2010).

A D-Optimal design algorithm was used to generate 4 blocks of 6 pairs of alternatives for respondents to evaluate (Xu, 2002). The conjoint exposure included combinations of a home, a vehicle and a vacation club as well as the choice to select none of the combinations over different time horizons ranging from 3 to 30 years. Beyond the conjoint design, a seven-point anchored rating scale was used for the evaluation of the overall concept to enhance the precision of utility calculation. Segment clusters based on concept evaluation were created using a variant of the K-means procedure known as the HowardHarris algorithm (Howard \& Harris, 1966). While segmentation was an important part of the original study, this analysis focuses solely on concept evaluation in the aggregate. Given that the research objective is to identify items to enhance interest in lifestyle bundles, the original responses, which directly addressed these questions, were analyzed and results were presented in the form of a histogram. The data cube was stored as an SPSS file.

\section{Sample and Data Collection Plan}

The sample parameters were established to include US consumers aged 18 and older with an annual household income equal to or greater than $\$ 150,000$. Additional screening criteria included sole or shared decision-making for major capital purchases. The study sample was drawn from over 3 million participants in the Greenfield Online (GOL) managed Internet panel. A double opt-in protocol as part of the self-selection process was initiated through personalized email invitations. Participants were compensated for their involvement through sweepstakes entry and nominal remuneration. Data was collected by GOL and stored in an SPSS database.

The plan was designed to generate 600 completed surveys to allow for necessary statistical analysis at sub-group levels such as age, gender and household income. The survey spanned 25 minutes in average length, which was five minutes longer than the pre-tested duration, and posed several challenges. As many as 789 participants abandoned the survey, which was higher than expected even at the chosen income level. The survey was left open for several additional days, which amounted to 8 total days for survey completion, to generate the necessary completed surveys. There was a gender response imbalance, with $80 \%$ female and $20 \%$ male participation, during the first few days of data collection, which pointed to the possibility of weighting techniques to be introduced. However, the gender gap closed to $65 \%$ female and $35 \%$ male, which was much closer to normative response levels for the tested income ranges, by the time the survey drew to a close. As a result, no weighting protocols were applied. Fifty-nine percent of the sample claimed household incomes ranging from $\$ 150,000$ to $\$ 199,999,29 \%$ claimed household incomes from $\$ 200,000$ to $\$ 299,000$, and $10 \%$ claimed household incomes from $\$ 300,000$ to $\$ 999,999$. Two percent of the sample claimed household incomes of $\$ 1,000,000$ and higher.

While plan budget had the greatest impact on the final number of completed surveys, a number of design criteria were considered when determining the final sample. Issues included the ability to ensure proper sample representation and manage sample error while completing a wide range of statistical analyses (Alreck \& Settle, 1995; Gay \& Diehl, 1992; Krejcie \& Morgan, 1970). Any actions taken to fulfill the plan such as sending out additional invitations to targeted groups followed generally accepted industry protocols.

\section{Results}

The focus of this analysis is to share the reactions of the respondents for enhancing the concept of the lifestyle bundle. The initial step in this process is to document the baseline response from the original research. Figure 1 identifies the distribution of ratings made for concept consideration. Almost one in five respondents rated the concept at 5, 6 or 7 in a 7-point scale. Adding an item "might or might not consider" rating of 4 to the prior group, the total responses increase to $46 \%$. Fifty-six percent of the respondents rated the concept at 3 or lower. It is important to note that, among those rating the concept at 3 or lower, almost $9 \%$ claimed to know someone else who might be interested in the concept. 


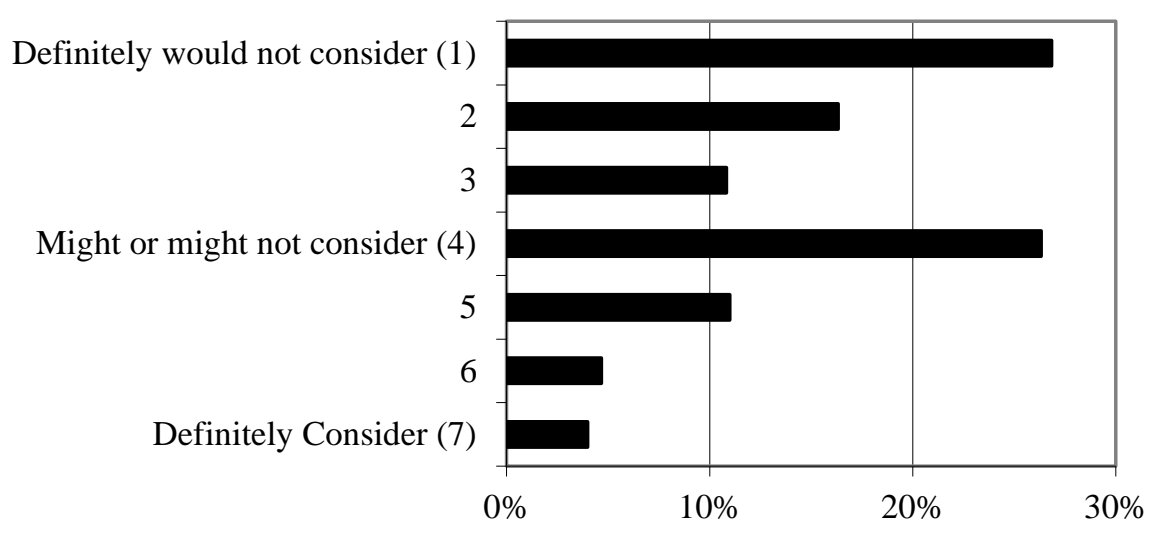

Fig. 1. Overall Concept Rating

Those who rated the concept between 4 and 7 identified the ability to "work with a single point of contact," "allows expression of lifestyle," and "convenience" as the three leading reasons for considering the concept in a positive light. Figure 2 illustrates the top mentioned reasons for considering the concept.

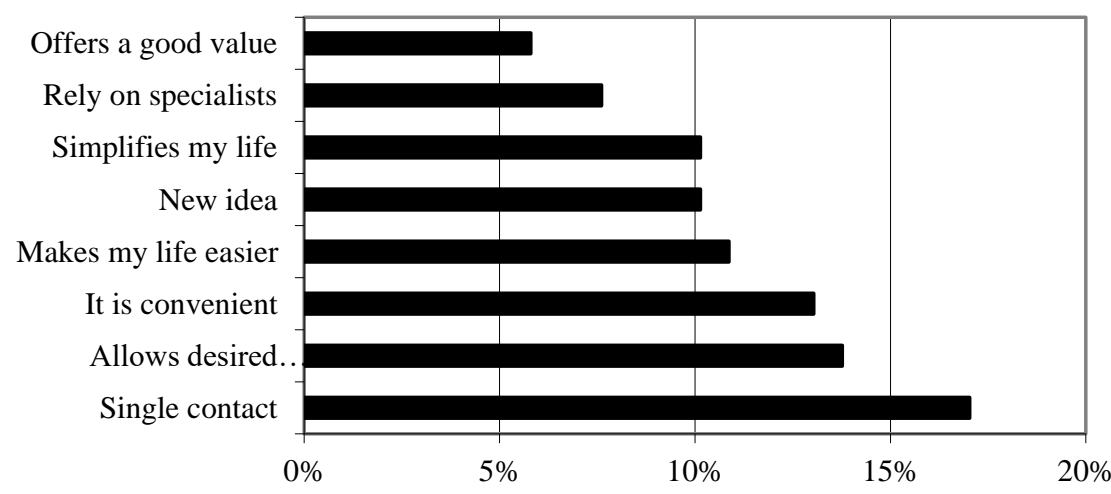

Fig. 2. Top Mention - Reason for Purchase Consideration

The novelty of the concept also contributed to its consideration by respondents. Figure 3 illustrates the level of uniqueness associated with the concept among those rating the concept at 4 or higher.

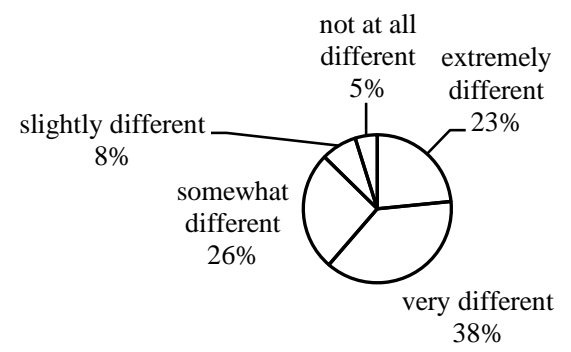

Fig. 3. Uniqueness among Those Who Support Concept 
Among those who rated the concept at 4 or higher, products and services were identified that would contribute to increasing their concept consideration rating beyond the current level. Figure 4 illustrates that insurance for the vehicle and home are the top rated enhancements to the concept to increase its consideration. Note that figure 4 is not based on top mention but instead includes multiple mentions.

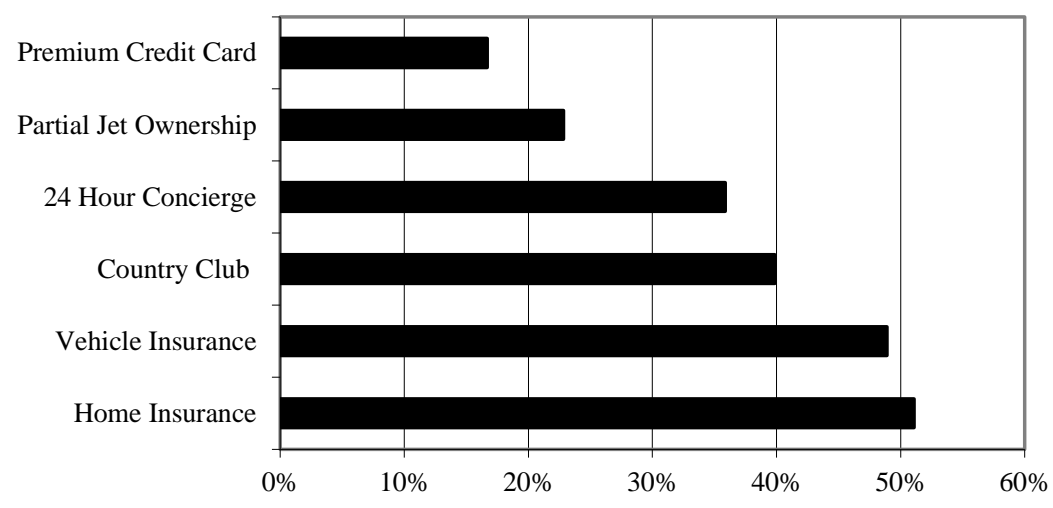

Fig. 4. Additions to Concept to Increase Consideration among Supporters

Among those who did not express interest in the concept by rating it at 3 or lower, the primary reasons for a lack of interest included a desire to retain the ability to make individual decisions within each category and not being in the market (currently shopping) for one or more of the products. Figure 5 illustrates the top mentioned reasons for rating the concept 3 or lower.

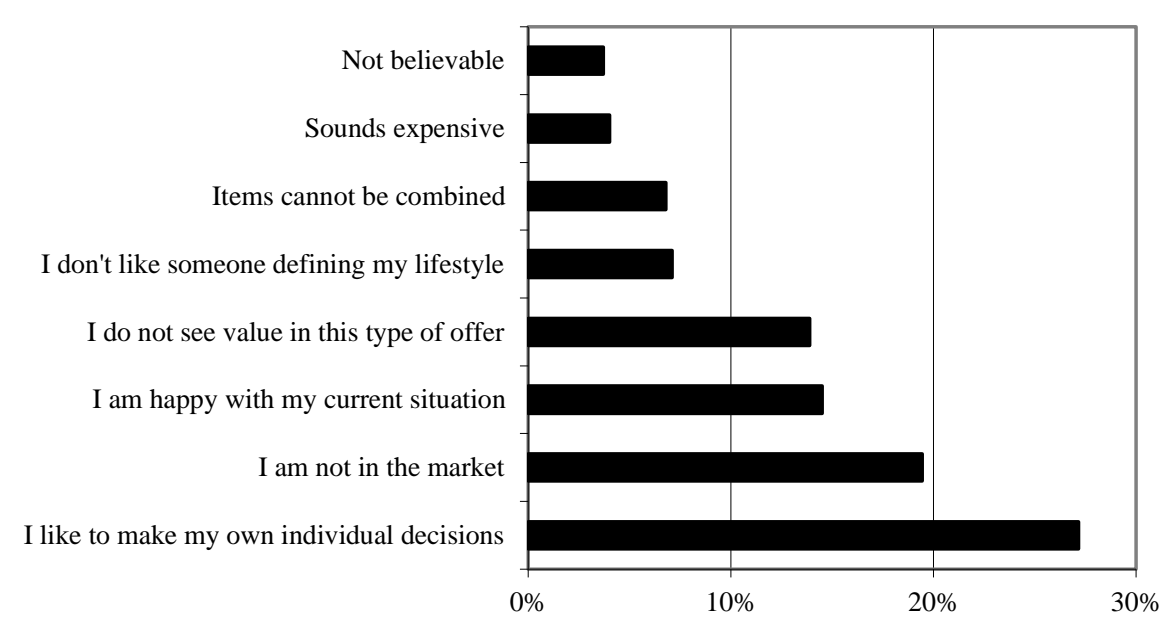

Fig. 5. Top Reasons Not Interested in Concept

Among those rating the concept at 3 or lower, $24.7 \%$ of the respondents mentioned that deleting the vacation club from the bundle would increase their concept interest to 4 or higher. Figure 6 illustrates the effect of deleting each of the bundle elements. 


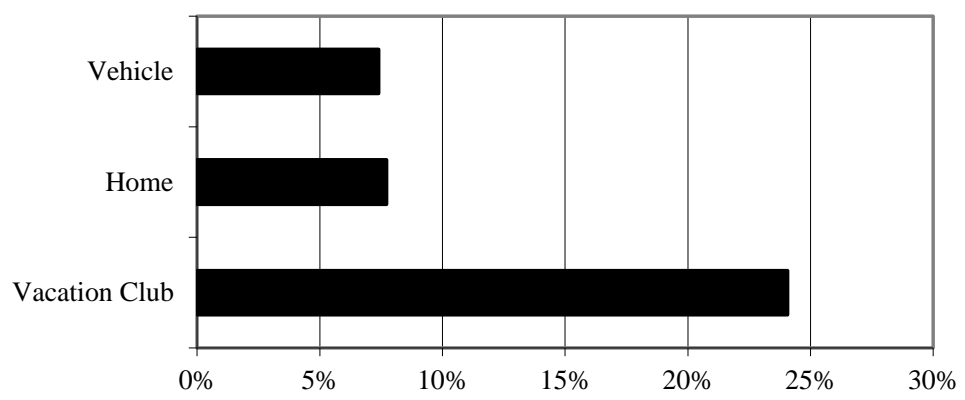

Fig. 6. Deletions to Concept to Increase Consideration among Detractors

A small number of individuals who rated the concept at 3 or lower identified additions to the concept that would contribute to increasing their consideration rating to 4 or higher. Figure 7 illustrates partial jet ownership and country club membership were the leading additions followed by home and vehicle insurance.

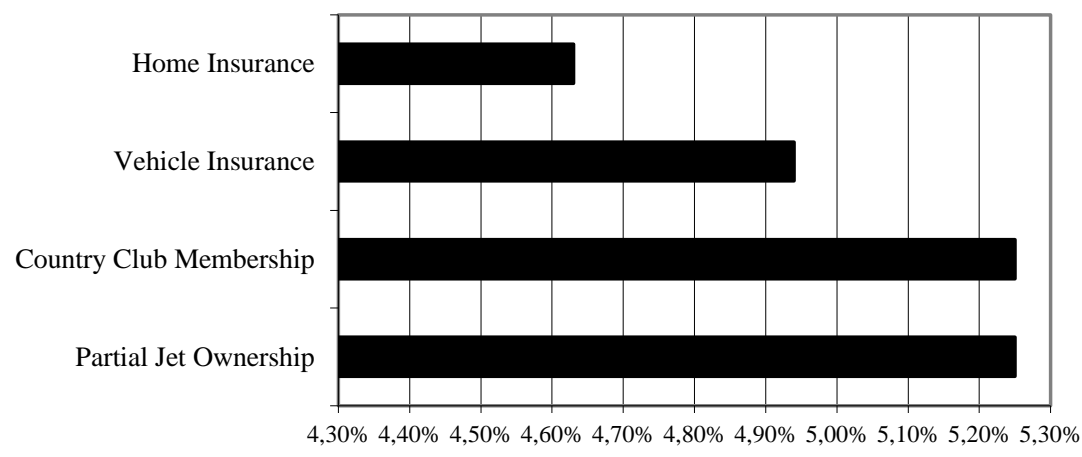

Fig. 7. Additions to Concept to Increase Consideration among Detractors

\section{Conclusions}

The original lifestyle bundle research identified a non-trivial level of interest in a home, vehicle and vacation club bundle. This paper identifies modifications to the bundle that would increase consumer interest among rejecters and supporters of the lifestyle bundle concept. While novelty was acknowledged as a reason for interest in the lifestyle bundle concept, it was clear that utility derived from convenience and lifestyle expression were the driving factors for many concept supporters. Those rejecting the concept also noted these attributes as influencing their opinions. Elimination of vacation club offer as part of the lifestyle bundle would have the greatest impact on increasing the rating of respondents who had rejected the concept. Adding home and vehicle insurance to the lifestyle bundle would have the greatest impact on enhancing the value proposition for those supporting the concept. Clearly, most concept evaluators, whether approving or disapproving, were able to view lifestyle as a product. That reality presents marketers with many interesting possibilities.

Future work on this topic should include applying conjoint testing protocols to enhanced lifestyle bundles based on the criteria identified in the "Results" section of this paper. Different consumer segments should be studied with alternative lifestyle bundles. Ultimately, specific brand and price bundles should be tested. 


\section{References}

Alrick, P. \& Settle, R. (1995). The Survey Research Handbook: Guidelines and Strategies for Conducting a Survey, $2^{\text {nd }}$ edition. Chicago: Irwin Professional Publishing.

Freeman, K., Nolan, G., Tyson. J., Lewis, K., Griefield, R. \& Gulati, R. (2004). How CEO's manage growth agendas. Harvard Business Review, (82), 124-132.

Gaeth, G., Levin, I., Chakraborty, G. \& Levin, A. (1990). Consumer evaluation of multi-product bundles: An information integration analysis. Marketing Letters, (1), 47-57.

Gay, L. \& Diehl, P. (1992). Research Methods for Business and Management. New York: Macmillan.

Gwin, C. \& Gwin, C. (2003). Product Attributes Model: A Tool for Evaluating Brand Positioning. Journal of Marketing Theory and Practice, (2), 30-42.

Howard, N., and Harris, B., (1966). A Hierarchical Grouping Routine, IBM 360/65 FORTRAN IV Program, University of Pennsylvania Computer Center Publication, 5-12.

Krejcie, R. \& Morgan, D. (1970). Determining sample size for research for research activities. Educational \& Psychological Measurement, (30), 607-610.

Levens, M. (2016). Marketing: Defined, Explained, Applied $3^{\text {rd }}$ edition. New York: Pearson Education.

Levens, M. (2010). Lifestyle Bundles: A Between-Category Product Evaluation Study of Affluent Consumer Behavior. Journal of Applied Marketing Theory, (1), 45-66.

Oppewal, H. \& Holyoake, B. (2004). Bundling and retail agglomeration effects on shopping behavior. Journal of Retailing and Consumer Services, (11), 61-74.

Raghavarao, D., Wiley, J. \& Chitturi, P. (2010). Choice-Based Conjoint Analysis Models and Design. Boca Raton: CRC Press.

Shocker, A., Bayus, B. \& Kim, N. (2004). Product complements and substitutes in the real world: the relevance of other products. Journal of Marketing, (1), 28-40.

Warsh, D. (1989). How selfish are people - really? Harvard Business Review, (67), 26-34.

$\mathrm{Xu}, \mathrm{H}$. (2002). An Algorithm for Constructing Orthogonal and Nearly-Orthogonal Arrays. Technometrics, (4), 356-368. 